\title{
Comparative integromics on FZD7 orthologs: Conserved binding sites for PU.1, SP1, CCAAT-box and TCF/LEF/SOX transcription factors within 5'-promoter region of mammalian FZD7 orthologs
}

\author{
MASUKO KATOH ${ }^{1}$ and MASARU KATOH ${ }^{2}$ \\ ${ }^{1}$ M\&M Medical BioInformatics, Hongo 113-0033; ${ }^{2}$ Genetics and Cell Biology Section, \\ National Cancer Center Research Institute, Tokyo 104-0045, Japan
}

Received November 22, 2006; Accepted December 27, 2006

\begin{abstract}
Canonical WNT signals are transduced through Frizzled (FZD) family receptor and LRP5/LRP6 co-receptor to upregulate MYC, CCND1, FGF20, JAG1, WISP1 and DKK1 genes, while non-canonical WNT signals are transduced through FZD family receptor and PTK7/ROR2/RYK coreceptor to activate RHOA/RHOU/RAC/CDC42, JNK, PKC, NFAT and NLK signaling cascades. FZD7, expressed in the normal gastrointestinal tract, is upregulated in esophageal cancer, gastric cancer, colorectal cancer, and hepatocellular carcinoma. Here, chimpanzee $F Z D 7$ and cow $F z d 7$ genes were identified and characterized by using bioinformatics (Techint) and human intelligence (Humint). Chimpanzee FZD7 and cow Fzd7 genes were identified within NW_001232110.1 and AC173037.2 genome sequences, respectively. Chimpanzee FZD7 and cow Fzd7 showed 100\% and 97.2\% total-aminoacid identity with human FZD7. All of the nine amino-acid residues substituted between human FZD7 and human FzE3 were identical to those of human FZD7 in chimpanzee, cow, mouse and rat FZD7 orthologs. Functional analyses using FzE3 with multiple cloning artifacts and/or sequencing errors are invalid. FZD7 orthologs were seven-transmembrane proteins with extracellular Frizzled domain, leucine zipper motif around the 5th transmembrane domain, and cytoplasmic DVL- and PDZ-binding motifs. Ser550 and Ser556 of FZD7 orthologs were putative aPKC phosphorylation sites. Dimerization and Ser550/556 phosphorylation were predicted as regulatory mechanisms for the signaling through FZD7. Transcriptional start site of human FZD7 gene was 735-bp upstream of NM_003507.1 RefSeq 5'-end. In addition to gastrointestinal cancer, hepatocellular cancer and pancreatic cancer, human FZD7 mRNAs were expressed in blastocysts, undifferentiated embryonic stem (ES) cells, ES-derived endodermal progenitors,
\end{abstract}

Correspondence to: Dr Masaru Katoh, Genetics and Cell Biology Section, National Cancer Center Research Institute, 5-1-1 Tsukiji, Chuo-ku, Tokyo 104-0045, Japan

E-mail: mkatoh-kkr@umin.ac.jp

Key words: WNT, Frizzled, theoretical biology, stem cell signaling network, integrome network, systems medicine
ES-derived neural progenitors, fetal cochlea, retinal pigment epithelium, olfactory epithelium, regenerating liver, and multiple sclerosis. Comparative genomics analyses revealed that the binding sites for PU.1, SP1/Krüppel-like, CCAATbox, and TCF/LEF/SOX transcription factors were conserved among 5'-promoter regions of mammalian FZD7 orthologs.

\section{Introduction}

Cross-talk of the WNT signaling pathway and FGF, Notch, Hedgehog and BMP/Nodal/TGFß signaling pathways constitute the stem-cell signaling network, which is implicated in embryogenesis and adult tissues homeostasis (1-13). Canonical WNT signals are transduced through Frizzled (FZD) family receptor and LRP5/LRP6 co-receptor to upregulate $M Y C$, CCND1, FGF20, JAG1, WISP1 and DKK1 genes (14-24), while non-canonical WNT signals are transduced through the FZD family receptor and PTK7/ROR2/RYK co-receptor to activate RHOA/RHOU/RAC/CDC42, JNK, PKC, NFAT and NLK signaling cascades (25-30). WNT signals are contextdependently transduced to canonical and non-canonical signaling cascades.

We previously reported molecular cloning and characterization of human FZD7 (31), which showed six amino-acid substitutions with human FzE3 (32). We then identified and characterized rat $F z d 7$ gene (33). FZD7 is upregulated in gastric cancer $(31,34)$, esophageal cancer (32), colorectal cancer $(31,35)$, and hepatocellular carcinoma (36). Here, chimpanzee FZD7 and cow $F z d 7$ genes were identified and characterized by using bioinformatics (Techint) and human intelligence (Humint). Chimpanzee FZD7 and cow Fzd7 genes were identified within NW_001232110.1 and AC173037.2 genome sequences, respectively. Comparative proteomics analyses on FZD7 orthologs were then performed. In silico expression analyses revealed FZD7 expression in human embryonic stem (ES) cells. In addition, comparative genomics analyses on FZD7 promoter region revealed conserved transcription factor binding sites within 5'-promoter region of mammalian FZD7 orthologs.

\section{Materials and methods}

Identification and characterization of chimpanzee and cow FZD7 orthologs. Chimpanzee and cow genome sequences 
A
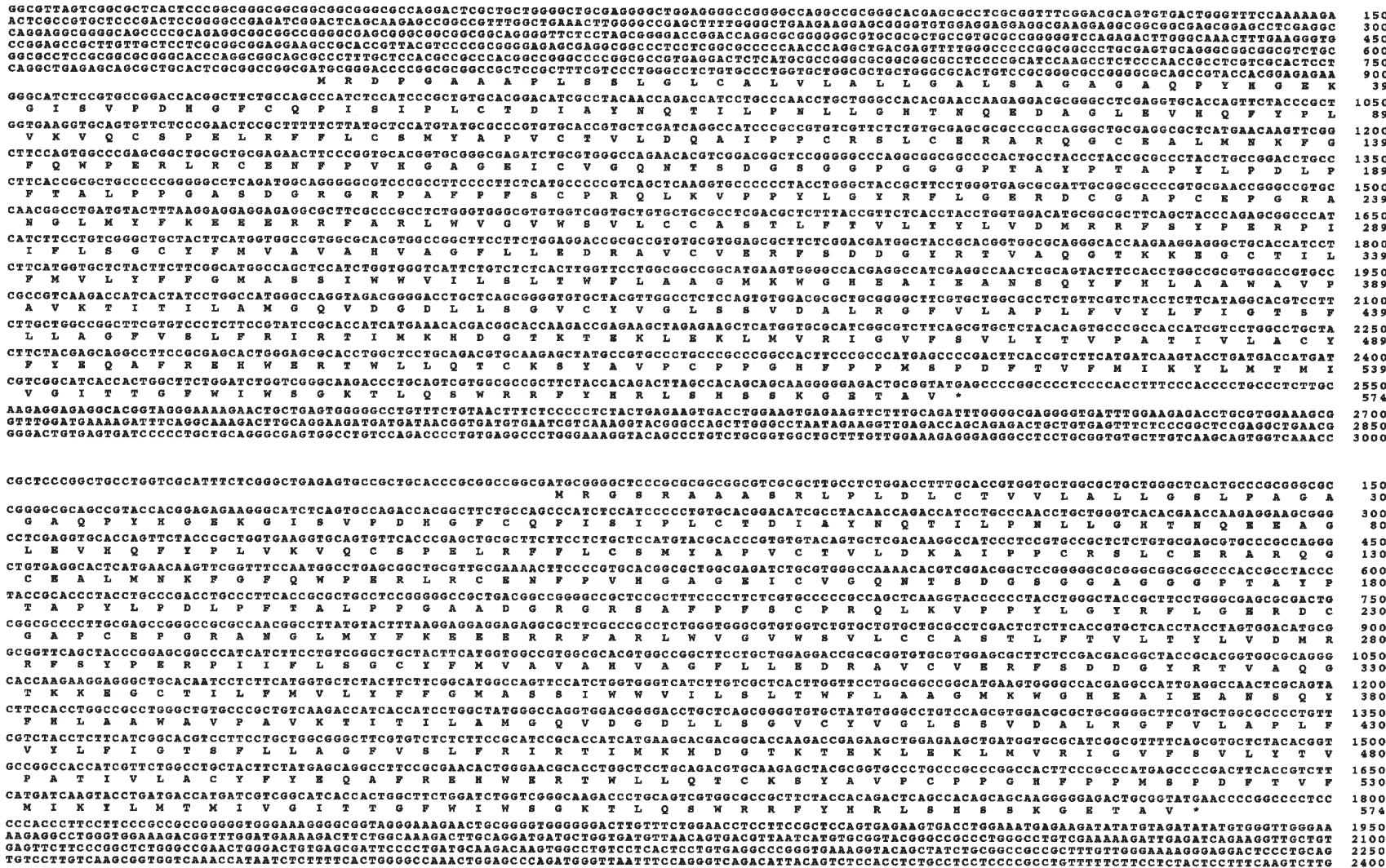

Figure 1. Chimpanzee and cow FZD7 orthologs. (A), Chimpanzee FZD7 complete CDS. Nucleotide position 1-3000 is shown. Nucleotides and amino-acid residues are numbered on the right. (B), Cow FZD7 CDS. Nucleotide position 1-2400 is shown. Nucleotides and amino-acid residues are numbered on the right.

homologous to human FZD7 were searched for with BLAST programs as described previously (37-39). Exon-intron boundaries were determined based on the consensus sequence of exon-intron junctions ('gt ... ag' rule of intronic sequence) and codon usage within the coding region as described previously (40-42). Complete coding sequence (CDS) of chimpanzee FZD7 or cow Fzd7 was determined by assembling exonic region(s).

Comparative proteomics analyses. The domain architecture of FZD7 orthologs was analyzed by using RPS-BLAST and PSORT II programs.

In silico expression analyses. Expressed sequence tags (ESTs) derived from human FZD7 were searched for by using the BLAST programs as described previously (43-45). The sources of human ESTs were listed up for in silico expression analyses.

Comparative genomics analyses. Human genome sequences around the FZD7 gene was compared with chimpanzee, cow, mouse and rat genome sequences to identify evolutionarily conserved regions. Binding sites for transcription factors were then searched for as described previously.

\section{Results}

Transcriptional start site of human FZD7. FZD7 gene at human chromosome $2 \mathrm{q} 33.1$ is located within human genome sequence AC069148.6 as previously reported (33). BLAST programs using human genome sequence around the FZD7 gene revealed that several FZD7 ESTs were transcribed from more upstream position than the 5'-end of FZD7 RefSeq (NM_003507.1). CD673704.1 EST was transcribed from 735-bp upstream position, CN288787.1 EST from 706-bp upstream position, CN370065.1 EST from 699-bp upstream position, and CN370066.1 EST from 654-bp upstream position. Based on these facts, it was concluded that the transcriptional start site of human FZD7 gene was 735-bp upstream of NM_003507.1 RefSeq 5'-end.

Chimpanzee FZD7 and cow Fzd7 genes. BLAST programs using human FZD7 complete CDS revealed that chimpanzee FZD7 gene was located within NW_001232110.1 genome sequence. FZD7 gene without intron corresponded to the nucleotide position 33170705-33175279 of NW_001232110.1. Complete CDS of chimpanzee FZD7 was then determined. Genetyx program revealed that nucleotide position 785-2509 was the coding region. Chimpanzee FZD7 gene was found to encode a 574-amino-acid protein (Fig. 1A)

BLAST programs revealed that cow Fzd7 gene was located within AC173037.2 genome sequence. CDS of cow Fzd7 was next determined. Genetyx program revealed that nucleotide position 62-1786 was the coding region. Cow $F z d 7$ gene was found to encode a 574-amino-acid protein (Fig. 1B).

Comparative proteomics analyses on FZD7 orthologs. Chimpanzee FZD7 and cow Fzd7 showed 100\% and 97.2\% total-amino-acid identity with human FZD7, respectively. Among nine amino-acid substitutions between human FZD7 (31) and human FzE3 (32), Ala8, Leu15, Arg201, Leu308, 


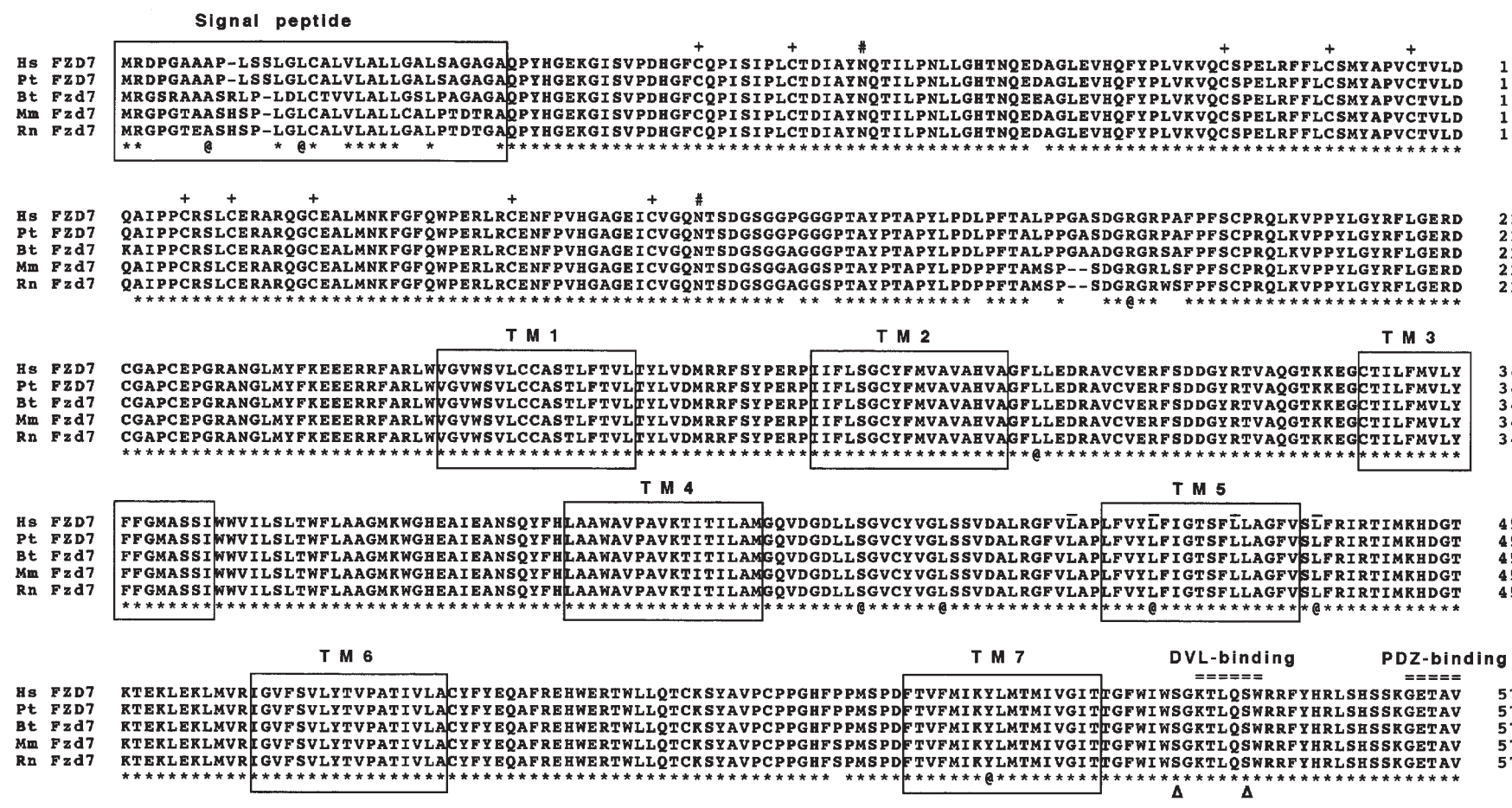

FzD7 CAPCEPCRANGLYFKEE

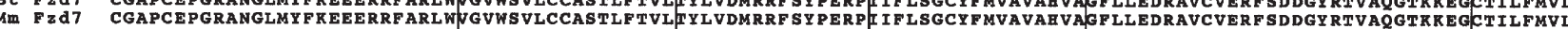

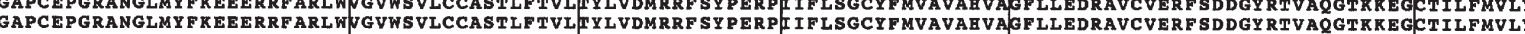

T M 4

T M 5

Figure 2. Mammalian FZD7 orthologs. Hs, human; Pt, chimpanzee; Bt, cow; Mm, mouse; Rn, rat. Signal peptide and seven-transmembrane domains (TM1-TM7) are boxed. Amino-acid residues are numbered on the right. Conserved Cys residues (cross) and Asn-linked glycosylation sites (sharp) within the N-terminal extracellular Frizzled region, leucine zipper motif around the TM5 domain (over line), DVL-binding and PDZ-binding motifs within the C-terminal cytoplasmic region (double over line) are shown above the alignment. Ser550 and Ser556 around the DVL-binding motif (open arrow head) and conserved aminoacid residues (* or @) are shown below the alignment. Locations of nine amino-acid substitutions between human FZD7 and human FzE3 (@) are also shown.

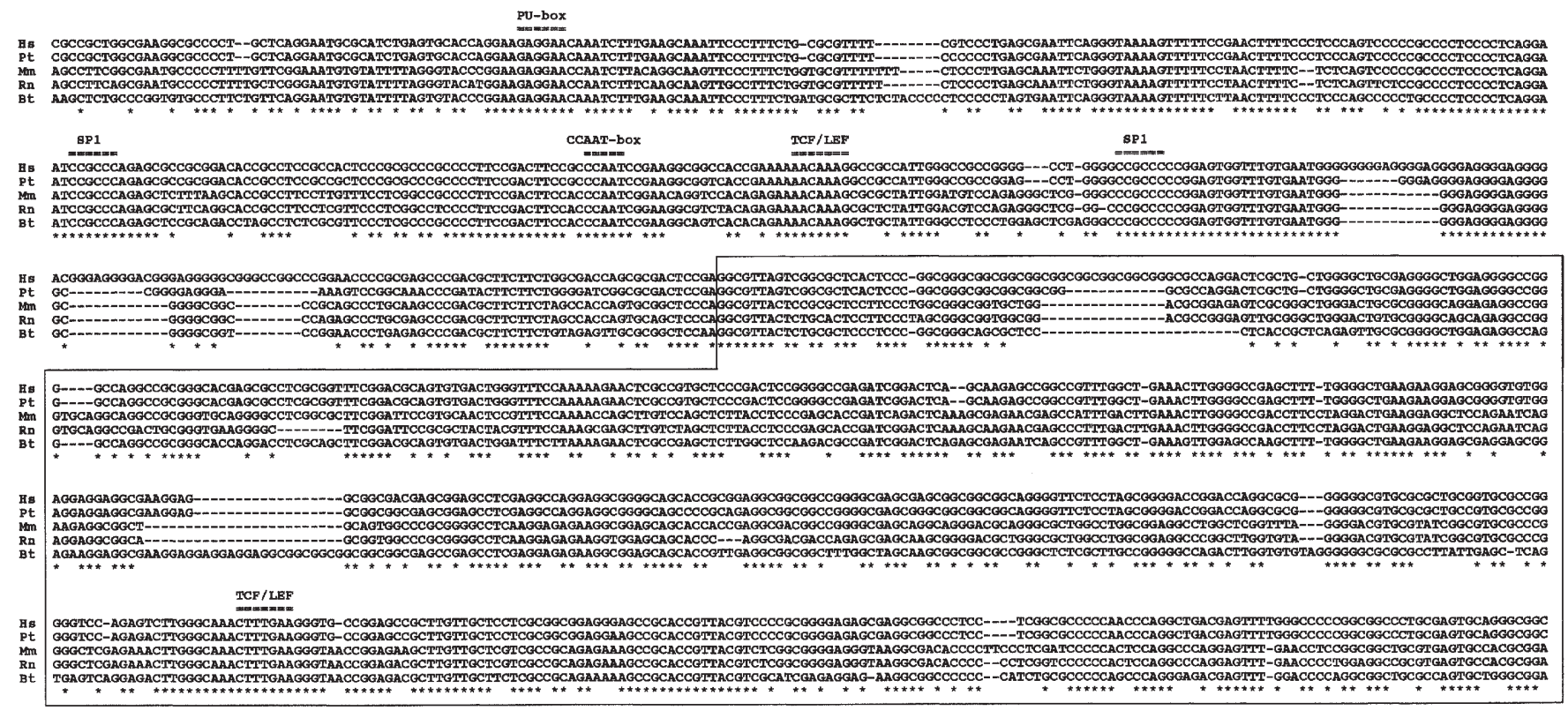

Figure 3. Mammalian FZD7 promoters. Hs, human; Pt, chimpanzee; Bt, cow; Mm, mouse; Rn, rat. Region corresponding to human FZD7 exon is shown by an open box. Conserved PU.1-, SP1-, CCAAT box-, and TCF/LEF/SOX-binding sites are shown by double overlines.

Ser408, Leu415, Leu433, Leu447 and Tyr534 corresponding to human FZD7 were conserved among mammalian FZD7 orthologs (Fig. 2). These facts indicate that 9 amino-acid substitutions in FzE3 are caused by sequencing errors and/or cloning artifacts.

FZD7 orthologs were seven-transmembrane proteins with extracellular Frizzled domain and, leucine zipper motif around the 5th transmembrane domain, and cytoplasmic DVL- and PDZ-binding motifs (Fig. 2). Asn63 and Asn164 within the $\mathrm{N}$-terminal extracellular region of FZD7 orthologs were Asnlinked glycosylation sites. Ser550 and Ser556 around the DVLbinding motif of FZD7 orthologs were very similar to Ser554 and Ser560 of Drosophila Frizzled, which are phosphorylated by human aPKC (46). 
In silico expression analysis on human FZD7. Expression of human FZD7 mRNAs were detected in blastocysts, ES cells in undifferentiated state, ES cells differentiated to endodermal progenitors, ES cells differentiated to neural progenitors, fetal cochlea, retinal pigment epithelium, olfactory epithelium, regenerating liver, multiple sclerosis, and a variety of cancer, such as gastric cancer, colorectal cancer, pancreatic cancer, head/neck tumors, adrenal cortex carcinoma, lymphoma, osteosarcoma, melanoma and germ cell tumors.

Comparative genomics analyses on FZD7 orthologs. Human FZD7, chimpanzee FZD7 and cow $F z d 7$ genes are located within AC069148.6, NW_001232110.1 and AC173037.2 genome sequences, respectively, as mentioned above. Mouse $F z d 7$ and rat $F z d 7$ genes are located within AC132574.3 and AC136379.2 genome sequences, respectively, as previously reported (33). The 5'-promoter regions of mammalian FZD7 orthologs were aligned to search for the conserved transcription factor-binding sites. PU.1-, SP1-, CCAAT box-, and $\mathrm{TCF} / \mathrm{LEF} / \mathrm{SOX}$-binding sites within 5'-promoter regions of mammalian FZD7 orthologs were evolutionarily conserved (Fig. 3).

\section{Discussion}

Comparative integromics analyses on FZD7 orthologs were performed in this study. Chimpanzee FZD7 was identified within NW_001232110.1 genome sequence, while cow Fzd7 gene within AC173037.2 genome sequence. Chimpanzee FZD7 and cow $F z d 7$ genes were found to encode 574-amino-acid protein showing $100 \%$ and $97.2 \%$ total-amino-acid identity with human FZD7, respectively (Fig. 1).

FZD7 orthologs were seven-transmembrane proteins with extracellular Frizzled domain, leucine zipper motif around the 5th transmembrane domain, and cytoplasmic DVL- and PDZbinding motifs. Ser550 and Ser556 of FZD7 orthologs were putative aPKC phosphorylation sites (Fig. 2). Dimerization is necessary for the functional activation of seven-transmembrane G-protein-coupled receptors (47). Cytoplasmic C-terminal phosphorylation on Drosophila Frizzled by human aPKC is implicated in the inhibition of Frizzled signaling to the noncanonical WNT signaling pathway or planar cell polarity (PCP) signaling pathway (46). Together, these facts indicate that dimerization and Ser550/556 phosphorylation are important for the regulation of the signaling through FZD7.

All of the nine amino-acid residues substituted between human FZD7 and human FzE3 were identical to those of human FZD7 in chimpanzee, cow, mouse and rat FZD7 orthologs (Fig. 2), which clearly indicates that FzE3 is an aberrant cDNA with multiple sequencing errors and/or cloning artifacts. Because Leu433 and Leu447 are substituted to Phe433 and Phe447 in FzE3, leucine zipper motif around the 5th transmembrane domain is disrupted in FzE3 as previously pointed out $(33,34)$. Therefore, functional analyses using FzE3 are invalid.

Transcriptional start site of human FZD7 gene was 735-bp upstream of NM_003507.1 RefSeq 5'-end. In silico expression analyses revealed that human FZD7 mRNAs were expressed in blastocysts, undifferentiated ES cells, ES-derived endodermal progenitors, ES-derived neural progenitors, fetal cochlea, retinal pigment epithelium, olfactory epithelium, regenerating liver, and multiple sclerosis. Comparative genomics analyses revealed that the binding sites for PU.1, SP1/Krüppel-like, CCAAT-box, and TCF/LEF/SOX transcription factors were conserved among 5'-promoter region of mammalian FZD7 orthologs (Fig. 3). Human FZD7 mRNA is expressed in gastrointestinal tract and gastroenterological cancer (31-36), and mouse $F z d 7$ mRNA is expressed in stem/progenitor cells in colonic epithelium (48). Together, these facts indicate that FZD7 plays a key role for ES cells and gastrointestinal stem/progenitor cells to orchestrate the scenario of embryogenesis and tissue homeostasis, respectively.

\section{References}

1. Katoh M: $W N T$ and $F G F$ gene clusters. Int J Oncol 21: 1269$1273,2002$.

2. Garciadiego-Cazares D, Rosales C, Katoh M and ChimalMonroy J: Coordination of chondrocyte differentiation and joint formation by $\alpha 5 \beta 1$ integrin in the developing appendicular skeleton. Development 131: 4735-4742, 2004.

3. Katoh Y and Katoh M: Hedgehog signaling in gastric cancer. Cancer Biol Ther 4: 1050-1054, 2005.

4. Clevers H: Stem cells, asymmetric division and cancer. Nat Genet 37: 1027-1028, 2005.

5. Katoh M and Katoh M: FGF signaling network in the gastrointestinal tract. Int J Oncol 29: 163-168, 2006.

6. Katoh $\mathrm{M}$ and Katoh M: Bioinformatics for cancer management in the post-genome era. Technol Cancer Res Treat 5: 169-176, 2006.

7. Katoh M and Katoh M: Cross-talk of WNT and FGF signaling pathways at GSK3ß to regulate B-catenin and SNAIL signaling cascades. Cancer Biol Ther 5: 1059-1064, 2006.

8. Katoh Y and Katoh M: FGF signaling inhibitor, SPRY4, is evolutionarily conserved target of WNT signaling pathway in progenitor cells. Int J Mol Med 17: 529-532, 2006.

9. Katoh $M$ and Katoh M: NUMB is a break of WNT - Notch signaling cycle. Int J Mol Med 18: 517-521, 2006.

10. Katoh Y and Katoh M: WNT antagonist, SFRP1, is Hedgehog signaling target. Int J Mol Med 17: 171-175, 2006.

11. Katoh Y and Katoh M: Comparative genomics on BMP4 orthologs. Int J Oncol 27: 581-585, 2005.

12. Katoh M and Katoh M: CER1 is a common target of WNT and NODAL signaling pathways in human embryonic stem cells. Int J Mol Med 17: 795-799, 2006.

13. Katoh $\mathrm{Y}$ and Katoh M: Comparative integromics on BMP/GDF family. Int J Mol Med 17: 951-955, 2006.

14. Katoh M: Regulation of WNT signaling molecules by retinoic acid during neuronal differentiation in NT2 cells: threshold model of WNT action. Int J Mol Med 10: 683-687, 2002.

15. Katoh M: WNT2B: comparative integromics and clinical application. Int J Mol Med 16: 1103-1108, 2005.

16. Swain RK, Katoh M, Medina A and Steinbeisser H: Xenopus frizzled-4S, a splicing variant of Xfz4, is a context-dependent activator and inhibitor of Wnt/B-catenin signaling. Cell Commun Signal 3: 12, 2005.

17. Pinson KI, Brennan J, Monkley S, et al: An LDL-receptor-related protein mediates Wnt signalling in mice. Nature 407: 535-538, 2000 .

18. Kramps T, Peter O, Brunner E, et al: Wnt/wingless signaling requires BCL9/legless-mediated recruitment of pygopus to the nuclear B-catenin-TCF complex. Cell 109: 47-60, 2002.

19. Katoh $M$ and Katoh M: Identification and characterization of human $B C L 9 L$ gene and mouse Bcl9l gene in silico. Int J Mol Med 12: 643-649, 2003

20. Chamorro MN, Schwartz DR, Vonica A, et al: FGF20 and DKK1 are transcriptional target of $\beta$-catenin and FGF20 is implicated in cancer and development. EMBO J 24: 73-84, 2005.

21. Katoh $\mathrm{M}$ and Katoh M: Comparative genomics on FGF2O orthologs. Oncol Rep 14: 287-290, 2005.

22. Katoh M and Katoh M: Notch ligand, JAG1, is evolutionarily conserved target of canonical WNT signaling pathway in progenitor cells. Int J Mol Med 17: 681-685, 2006. 
23. Pennica D, Swanson TA, Welsh JW, et al: WISP genes are members of the connective tissue growth factor family that are upregulated in Wnt1-transformed cells and aberrantly expressed in human colon tumors. Proc Natl Acad Sci USA 95: 14717-14722, 1998.

24. Katoh Y and Katoh M: Comparative genomics on $D K K 1$ orthologs. Int J Oncol 27: 275-279, 2005.

25. Katoh M: WNT/PCP signaling pathway and human cancer. Oncol Rep 14: 1583-1588, 2005.

26. Yamaguchi TP, Bradley A, McMahon AP, et al: A Wnt5a pathway underlies outgrowth of multiple structures in the vertebrate embryo. Development 126: 1211-1223, 1999.

27. Lu X, Borchers AG, Jolicoeur C, et al: PTK7/CCK-4 is a novel regulator of planar cell polarity in vertebrates. Nature 430: 93-98, 2004.

28. Lu W, Yamamoto V, Ortega B, et al: Mammalian Ryk is a Wnt coreceptor required for stimulation of neurite outgrowth. Cell 119: 97-108, 2004.

29. Boutros M, Paricio N, Strutt DI, et al: Dishevelled activates JNK and discriminates between JNK pathways in planar polarity and wingless signaling. Cell 94: 109-118, 1998.

30. Dejmek J, Safholm A, Kamp Nielsen C, et al: Wnt-5a/Ca ${ }^{2+}$ induced NFAT activity is counteracted by Wnt-5a/Yes-Cdc42casein kinase Ia signaling in human mammary epithelial cells. Mol Cell Biol 26: 6024-6036, 2006.

31. Sagara N, Toda G, Hirai M, Terada M and Katoh M: Molecular cloning, differential expression, and chromosomal localization of human Frizzled-1, Frizzled-2, and Frizzled-7. Biochem Biophys Res Commun 252: 117-122, 1998.

32. Tanaka S, Akiyoshi T, Mori M, Wands JR and Sugimachi K: A novel frizzled gene identified in human esophageal carcinoma mediates APC/B-catenin signals. Proc Natl Acad Sci USA 95: 10164-10169, 1998.

33. Katoh $\mathrm{M}$ and Katoh $\mathrm{M}$ : Comparative genomics on FZD7 orthologs. Int J Mol Med 15: 1051-1055, 2005.

34. Kirikoshi $\mathrm{H}$, Sekihara $\mathrm{H}$ and Katoh $\mathrm{M}$ : Up-regulation of Frizzled-7 (FZD7) in human gastric cancer. Int J Oncol 19: 111-115, 2001 .

35. Vincan E: Frizzled/WNT signalling: the insidious promoter of tumor growth and progression. Front Biosci 9: 1023-1034, 2004.
36. Merle P, De la Monte S, Kim M, et al: Functional consequences of frizzled-7 receptor overexpression in human hepatocellular carcinoma. Gastroenterology 127: 1110-1122, 2004.

37. Katoh M: Paradigm shift in gene-finding method: from benchtop approach to desk-top approach. Int J Mol Med 10: 677-682, 2002.

38. Katoh M and Katoh M: Identification and characterization of human PRICKLE1 and PRICKLE2 genes as well as mouse Prickle1 and Prickle 2 genes homologous to Drosophila tissue polarity gene prickle. Int J Mol Med 11: 249-256, 2003.

39. Katoh $\mathbf{M}$ and Katoh $\mathbf{M}$ : Identification and characterization of human DAPPER1 and DAPPER2 genes in silico. Int J Oncol 22: $907-913,2003$.

40. Katoh $\mathbf{M}$ and Katoh $\mathrm{M}$ : Identification and characterization of human FMNL1, FMNL2 and FMNL3 genes in silico. Int J Oncol 22: 1161-1168, 2003.

41. Katoh $\mathbf{M}$ and Katoh $\mathbf{M}$ : Identification and characterization of human HES2, HES3, and HES5 genes in silico. Int J Oncol 25: 529-534, 2004.

42. Katoh $\mathrm{M}$ and Katoh M: Identification and characterization of human HESL, rat Hesl and rainbow trout hesl genes in silico. Int J Mol Med 14: 747-751, 2005

43. Katoh M: Molecular evolution of $W N T 2 B$ orthologs. Int J Oncol 26: 1135-1139, 2005.

44. Katoh $\mathrm{M}$ and Katoh $\mathrm{M}$ : Comparative genomics on FGF8, FGF17, and FGF18 orthologs. Int J Mol Med 16: 493-496, 2005.

45. Katoh Y and Katoh M: Comparative genomics on HHIP family orthologs. Int J Mol Med 17: 391-395, 2006.

46. Djiane A, Yogev S and Mlodzik M: The apical determinants aPKC and dPatj regulate Frizzled-dependent planar cell polarity in the Drosophila eye. Cell 121: 621-631, 2005.

47. Milligan G: G-protein-coupled receptor heterodimers: pharmacology, function and relevance to drug discovery. Drug Discov Today 11: 541-549, 2006

48. Gregorieff A, Pinto D, Begthel H, et al: Expression pattern of WNT signaling components in the adult intestine. Gastroenterology 129: 626-638, 2005. 